\title{
The importance of vertical buildings in perception and memorising the city
}

\author{
Rifat Alihodzic ${ }^{*}$, and Domen Zupančič² \\ ${ }^{1}$ University of Montenegro, Faculty of Architecture, Podgorica, Montenegro \\ ${ }^{2}$ University of Ljubljana, Faculty of Architecture, Slovenia
}

\begin{abstract}
Being aware of the surrounding we live in, among other things, means establishing of spatial relationships between oneself and the environment, equally important as relationship between oneself and others. Environment consists of facilities and space. Space, „gift by itself“, is defined by terrain topography, sky horizon, plants and animals. The architecture, as a profession, is interested in space created distinctively. Perception, as elementary process of consciousness and psychological life, deals with being conscious about something. In this case, physical structures that create a city. Psychological experience of urban environment is important factor having effect on perception, memorising and orientation in urban space. Gestalt psychology of perceiving is area applying to and significant for architecture either. The importance of vertical lies in its perceiving the gravitation, forming perceiving focus, landmark, for urban units and subunits to be memorised, creating spatial hierarchy and perception logics, remembering and orientation in space. This work analyses reasons for building upright with comparative analyses in their participation in space and on human psychology. This paper's purpose is to, using fundamental facts, show the importance of vertical buildings, not as a spatial use phenomenon, but also as significant phenomenon.
\end{abstract}

\section{Introduction}

When perceiving any object, the perception process defines its basic features which making that particular object distinctive from others. Perceptive process determines its visual identity.

Some of the most important features of each object are its shape, size, colour, structure of material, position in space and comprehension whether the object is dinamic or static. These are some basic features, and particularities of a certain object can show some other features, especially comparative ones, which distinguish them from similar objects in vicinity. [1]

In this paper, there will be only one feature of an object (in this case, a facility) examined and that is its vertical position in space.

\footnotetext{
*Corresponding author: rifata@ac.me
} 
Let's assume that there is a physical structure having dimensions $x, y, z$. It can be positioned in building area either horizontally or vertically. Cubic capacity of its space, its usagein other words, will be the same no matter on vertical or horizontal position.

However, participation in space in sense of perceiving, memorising and experiencing of that physical structure (form) won't be the same. That is because seeing something means giving it a place in a certain unit. [2]

Architectural facilities, in most cases, are not isolated as they seem to be in our minds when designing procedure takes place. Real space, in which they are incorporated, is always a context, no matter if it is a purely natural environment or built environment. That way, facilities are parts of a specific unit in a wider plan, but they can mostly be perceived in two ways: one of them represents a moment when the facility distinguishes because of its features in one's perception and the other one is when targeted focusing is achieved on purpose. Familiar fact, not only from Gestalt psychology, but also from experience, is that vertical facilities impose to our perception!

Important question when perceiving an object (form) basically is narrowed tothis: How to link what our perception with our memory, how to identify what we perceive and how to experience it? [3]

\section{Reasons for perceiving vertical and its importance}

For favouring vertical compared to horizontal in general perceiving, the gravitation is responsible, which people are accustomed to through the empathy. We should have in mind that vertical position is one of people's first experiences in space. It is not a coincidence that this is the first obligation of each designing act, when determining the way our eyes will perceive forces that the facility resists, the gravitation. Among countless directions of tridimensional space among which it is theoretically possible to move, only one direction has a gravitation as its feature: vertical. Vertical is a base and a measure for all other directions. [4]

From the geometrical point of view, there is no difference in up-down movement, but from the gravitation's point of view and designers' efforts, it surely has. The consciousness about that gives a special psychological relation towards vertical as a reference direction. What is important to perceive is that vertical position became a part of collective memory psychologically also. „Man in vertical“ means the presence of moral qualities and a wide range of other positive attributes.

Let's remind ourselves that, in construction, for example, a plumb line is used and every other direction is determined according to vertical direction. That is the case when the level is used and that is done comparing to already established vertical.

Vertical is the thing that determines horizontal direction so it serves as a symmetrical axis. Unlike vertical direction, horizontal is the level of moving in every possible direction without having the „obligation“ to gravitation. Horizontal level is a space of human existence and their activities. The house is assumed to be a concentrated vertical being. It rises up. The straight line dominates there. Plumb line left a seal of its sense and its balance. [5]

In favour to the comprehension of importance of vertical as a product of gravitation, there is an irresistable fact that there are no records of anyone wanting a bed or dining table on slope level. That is not only pragmatical thing, but also a thing of (dis)orientation which is obvious in stepping out of vertical. Our bodies and mind experience such situations as alarming ones. Nietzsche, who had influence of aestheitics of Hegel, Schopenhauer and Hartmann, noticed that. ,In architecture, the pride of a man, his triumph over the gravition, his will to power, assumesa visible form. Architecture is a sort of oratory power by means of form. " [6] 
Vertical is ,a bearer“ of perceptiontaking the position as a straight line. It goes downwards, up to some horizontal to be resistant to, and that is a straight line, or, in case of architectural facility, an Earth ground. The way that contact of the Earth and facility materializes, results in specific perceiving, depending on relationship established following the principle of ,visual statics“, which is not the same as engineering statics, dealing with the stability of the facility.

\section{Vertical facility as an urban landmark}

Generally, there are two ways for better orientation in space. They are defined by K. Linch. [7] One of them represents a kind of distionction, „landmark“. Other places are linked with this mark. More precise way that determines a place is a relation with some facility. It is absolutely logical that this kind of approach is more effective, especially when this is vertical. Abovementioned approaches represent fundamental elements of orientation.

Common things (facilities) do not attract our attention on regular basis, so the same applies for architecture. Psychology of perceiving teaches us that we are not able to concentrate to more things (architectural facilities) at the same time, so it is logical to focus on those that stand out, i.e. impose to our eyes. ,, In architecture, as in any other culture, our sense of how some things should be, develops from our experience. Each gesture that we make means something, but the meaning depends on the culture in which gesture is understood. Architecture is a gesture made with buildings. " [8]

Without any doubt, the most significant result of vertical facility is that it becomes an orientation agent, in other words, urban mark. Urban landmarks are required as the most reliable elements of perception, memorising and orientation in space. Each vertical physical structure produces more intensive visual energy than the horizontal one because it is in position of better transmitting of vector in every direction.

Being lost in space considers panic that creates the fear of the highest order. It is known that, if a person shows disorientation and fear, people say that they are „lost“. Where does it come from? ,, The infant sees himself as the center of the worldsurrounding him. “ [9] Their comprehension for a long time is that everything is subordinate to their needs and under their constant control. That is the thing that stays attached to humans for a long period of time and it is hard to get rid of it, so entire groups, nations and countries act from the point of ,own interests" through whose centricity manifests as a feature that affects motifs and points of view. However, it is essential to observe other subjects in one's life, having their own needs that need to be respected, so that correlation necessarily grades through respecting the other „someone else's centres“. At the end, it is realized that own „centre“ is only one on the heap. That way, centrical tendency is being complemented with „eccentric" one. „The eccentric spot will exhibit a dynamic tendency, probably a pull in thedirection of the centric position.. " [9]

Mutual action of two tendencies (centric and eccentric) makes the essence of human living. It is considered both in relations towards human beings and towards things that surround us. One of them, probably the most important one, is the space we live in.

Physicists of our time give us the explanation that universe is created from the energy field, so each thing, house, building, hill, represent a certain energy field. Being such, things are nothing but vector forces. In that particular sense, vectors are mentioned for the first time by psychologist K. Lewin in 1935. [10] Symmetrical frame, with vectors transmitted shaped as rays, represents typical centric composition. Establishing the relation between one's own centre and some spatial centre represents, according to previously mentioned logics, vectors transfer. These relations are multi-level ones, depending on the subject with which primary centre establishes relation. We accept that it exists in a dynamical sense, provoking perception. 
From what was previously said, conclusion is that a man sees himself as a centre of everything surrounding him. During their moving, this centre is also moving with them, and the rest of the world is seen as a sum of objects eccentrically towards them. Establishing such relationship does not depend only on man, as a primary centre, transmitting their „own vectors" towards the object, and also the objects transmit their vectors towards the beholder influencing their attention.

Vertical dimension serves as an area for visual thinking chiefly, and horizontal is the empire of activities. That moment favours vertical things as the objects of real perceiving and establishing the relationship of our "centre“ with centres of the objects around us.

Paying attention to followingcan contribute to explaining these attitudes: ,, In a dynamical sense, a tower standing in an empty field or a ceramic vase on a table are centres of visual energy sending their vectors in all directions and thereby surround themselves with a tensionloaded field that spreads and gradually peters out in empty space. " [11] So, domination of vertical in an empty space is the precondition of domination towards other objects that do not possess enough space „for transmitting vectors" so those able to act that way impose themselves as landmarks. This way, landmarks represent accents ofcertain places, or points that they are linked to, or their parts.

Exquisite effects of landmarks can be achieved in cases when the natural landmarksmatch inherited terrain topographically and are being marked with some facility. That kind of picture is common at Italian smaller places as well as world famous cities such as Istanbul (Fig.1). This topic is frequently used, especially when building fortifications and castles, where the defense is a primary function of such facilities' locating, so the perception catches landmarks produced, whichfrequently became symbols of many towns and significant marks on the roads, because among many hills the one with a particular facility is being standing out.
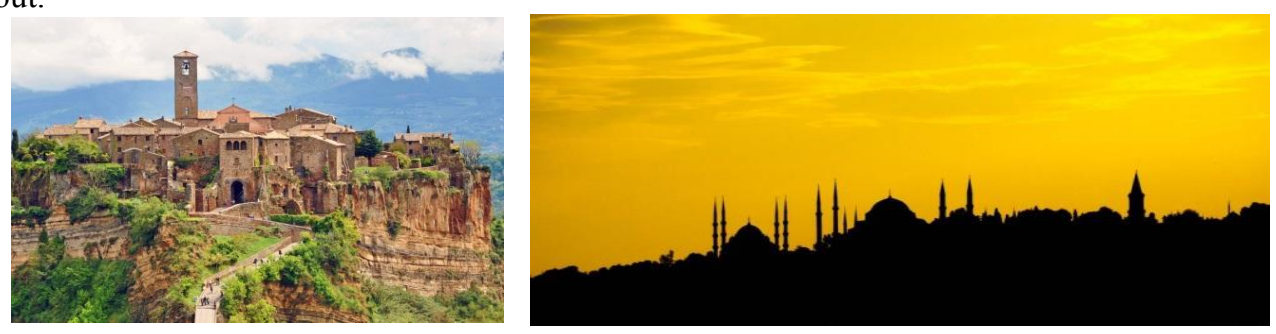

Fig.1.Italian village and silhouette of Istanbul. Matching both natural and a hierarchy created by building.

It is good to mention that, matching of landmark in certain situations can be a problem for an urban structure which, because of its ruined c hierarchy, can cause some difficulties in reading the space.

\section{Historical experiences with vertical buildings. Physical and spiritual landmarks}

To underline the place means to mark it by vertical. From the ancient times that moment was built in inside the human collective unconsciousness because the conscious experience suggests vertical as dominant one. 


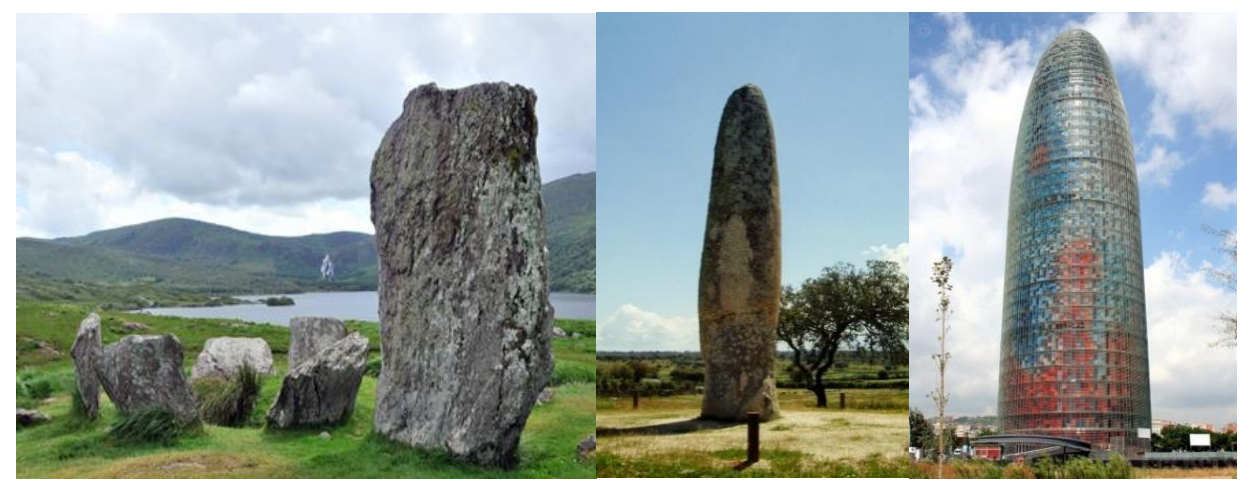

Fig.2. A stone menhires and Torre Agbar in Barcelona (arch. Jean Nouvel). The same goal in space no matter on the time of origin.

If we take a look at the building history and first human experiences of space, we inevitably meet phenomenonn of vertical, instinctively perceived as a strong orientation tool. Megalith structures of menhirs, dolmens and cromlechs, still having a dominant vertical, prove that. Doubtlessly, megalith structures have a feature of identifying and matching of physical and spiritual meanings, as well as all the sacral buildings created later on. Vertical is, above all, easy to notice and, comparing to horizontal, favours itself because it is easily perceived. Quantitatively, in the initial stage of human development, it gets a saint position in a sacral. [12]

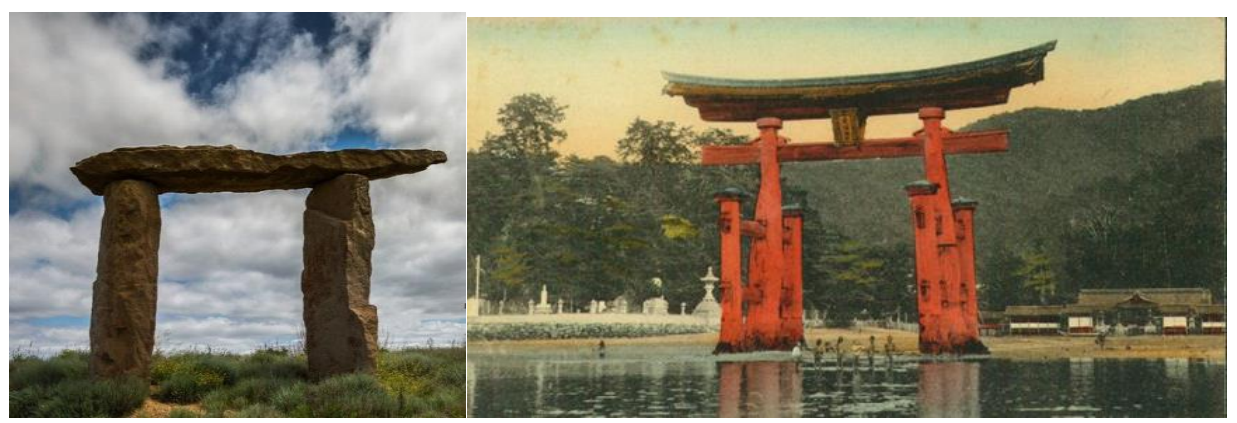

Fig.3. Simple spatial rock gate and contemporary Tori in Japan. The same idea in different temporal epochs.

If we have a glimpse at the history of certain vertical structures, we'll see its being constant in meaning and stability in form where only the envelope determines time of origin. (Fig.2,3).

Important moment in relation with vertical represents human notion of God, whose residence is connected to sky, so all sacred buildings are directed upwards and their perceiving structure has the effect of climbing. Sacral buildings, during the time, lose their prime, and are being replaced by those which, instead of God's power, manifest human power (Fig.4). 


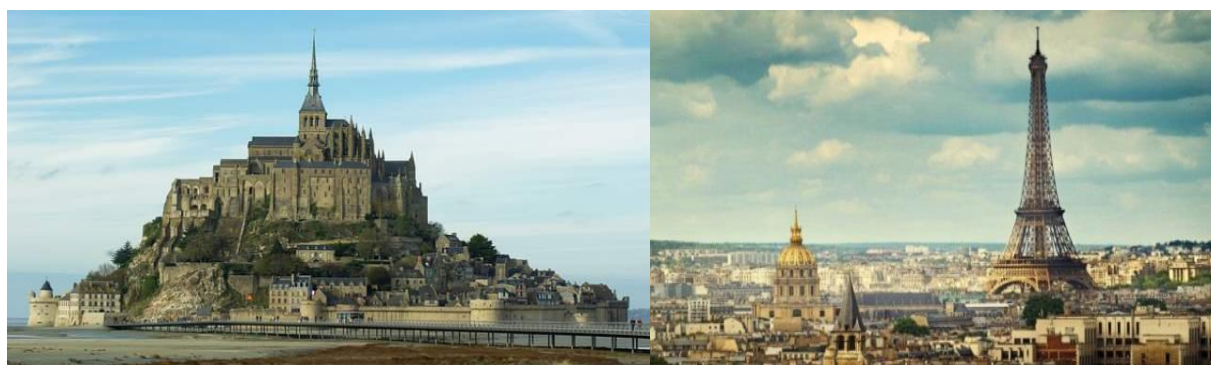

Fig.4. Mont Saint-Michel, vertical dedicated to God. Eiffel Tower, dedicated to the power of contemporary man.

From the very first marks in the space, over ziggurats and pyramides all through the total development of sacral buildings, irreplacable imortance of vertical is being sensed. So, importance of vertical was both a physical perceiving structure and a spiritual element required at sacral buildings, from historic point of view.

\section{Vertical facility as a manifestation of economical power and technological culture}

In human history, consuming society and economical power in its final (contemporary) phase show domination above spiritual and religious. That is not hard to notice through perceiving the city development where, up to 19th century, it was considered as perfectly normal and sacred, the rule that the highest facilities in space are those of sacral nature.

Impression created by vertical quantity estimates the height of an architectural facility and has own reasons within itself which have the notion of strong technological progress and manifestation of material power. Technological evolution is the thing that fascinates a modern man, so our civilisation considers it as almost natural and necessary process, just like biological one according to Darwin (Fig.5.).

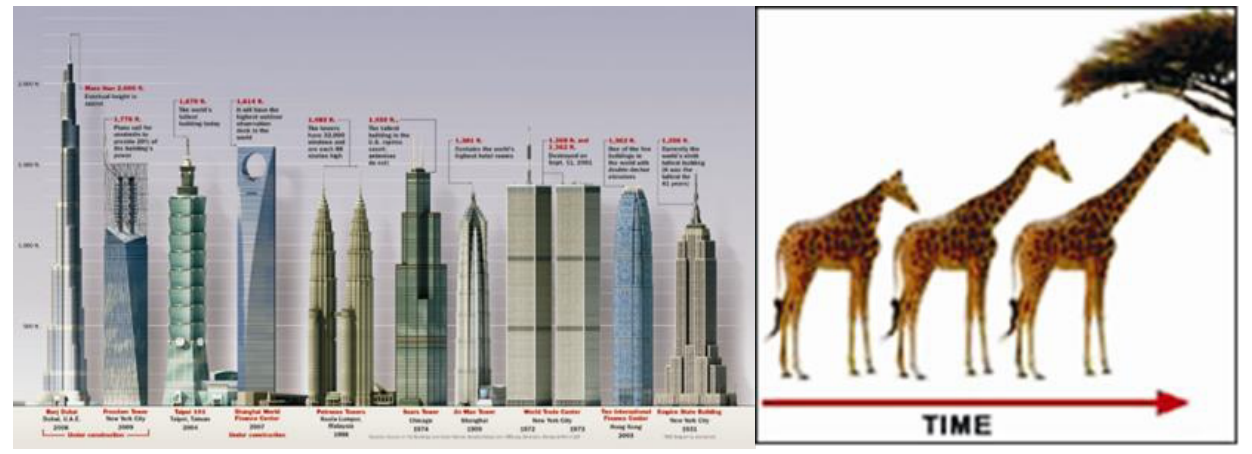

Fig.5. Each new, the highest building redefines the power scale. Similarity with Darwin's evolution theory.

Every new facility in the world, which changes scale of vertical quality, causes position redefinition, manifested in countries' power and that power is being manifested through multinational companies. If we make a comparison of the analyses based on quality of architectural facilities, by that, we can see that such system of evaluation ends only in the zone of professional circles, like Pritzker award and similar ones for instance. However, public opinion follows the fact that the new power centre will appear manifested by built vertical facility with greater curiosity and passion. 
Vertical size of an object, structure and facade appearing of architectural facility are measurements of technological improvement nowadays unlike the forms from previous period. That can be interpreted as growth of new creative and available energy, which is obvious in the connection of architectural expression and technological advantages of modern and ancient civilizations.

Every new facility in the world, which changes vertical qualitative scale causes redefinition of positions showing the strength of countries and their power, manifested through multinational compositions. As a comparison, no one has made a qualitative comparison of horizontal volumes at architectural facilities so far.

\section{Vertical facility as utilitarian component}

If we take a look at the building history, vertical facilities made from the requirements for space quantity as a primary reason are the youngest one and in less number.

Building siloes, water towers, bridges and other engineering constructions, as well as vertical buildings represents strictly utilitarian spatial-functional category, intensively appearing with industrial society development.
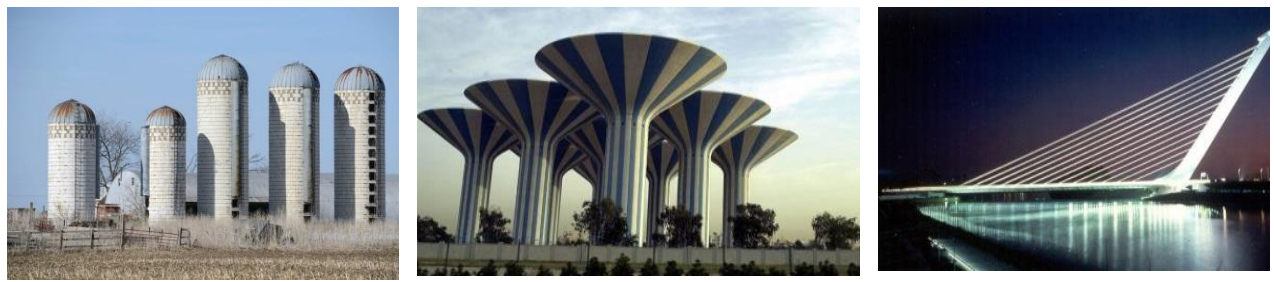

Fig.6. Siloes, water towers and bridges. Beside utilitarian importance, they take the role od landmark.

Even though these facilities mostly aren't primarily accentuating the space, but came out of practical purposes, they take over a bunch of attributes connected to the meaning in space. It is particularly obvious with water towers and bridges which manifest new technological era and economical power, that they started to be significant and possible city accents in urban designer's primary purpose (Fig.6).

Building of residential buildings, for selling or renting, represents typical examples of economically justified building which find their reasons for expensive urban-construction ground. However, in terms of humanity and residential conditions, this factor is dehumanisation of the way of living so our psychological experience doesn't recognize it as the nice one. The same can be applied to student dormitories, single people accomodation and similar facilities. If our starting point is that fundamental part of urban structure of the city contains $70-85 \%$ of buildings for residential purposes, then no one can neglect the occupying of space of this kind of facilities directed upwards at the expense of humanliving (Fig.6,7).

Business buildings for renting to other companies an in better position, while and the buildings meant for the purposes of one company, having its trademark are in best position. Then, they obtain a clear and precise meaning considering power manifestation of a specific owner, company that built that facility.

Therefore, it is good to say that dehumanisation of living space in business high towers is not less significant than the one in residential areas, because a man spends most of his everyday life at his work, being awake, while is his residential space meant only for night break and sleeping. 


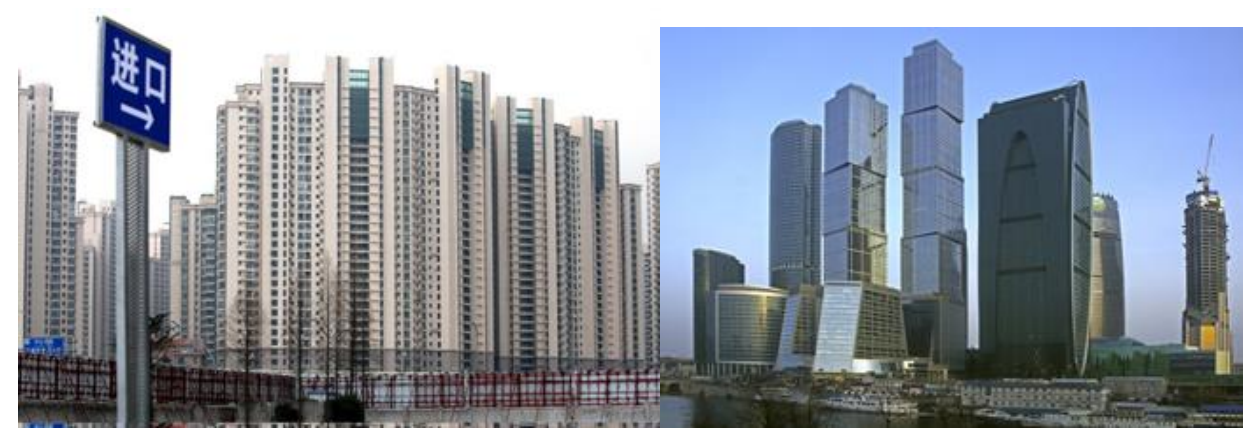

Fig.7. High residential and business buildings make significant landmarks.

No matter the goals and reasons for building and above mentioned facilities have their role in space, theycan be caught by an eye and significantly define beholder's experience. However, categorisation of total meaning of their function does not give them significance as well as to sacral buildings and those meant for culture and sport.

No doubt that in the second half of 20th and and in 21 st century, facilities of culture and sport along with business facilities dedicated to power and multinational companies, take the position over the facilities of sacral features.

\section{Conclusion}

Vertical and quantity of an object are the most important rules of perceiving organisation in space. [13] However, that alone is not the reason enough for completeness and perceiving the total experience. All things in process of perception carry the meaning even when those creating them do not have intention of sending a certain message. However, when designing performance aims for meaning, which the observer interprets, there can be made an effect upon it for sure. [14]

Basically, urban structure is made by landmarks built with a time residue and a requiring experience. When they establish themselves as such, they become the elements on whose basis can the whole urban picture be created. That happens when we create spatial correlation between them or we have graphically prepared structural scheme in front of them. By identification of one of such facilities can possibly reconstruct the whole urban picture. As the illustration of this claim, a part from tourist prospect of Paris was used for this purpose, where only landmarks with discreetly emphasised Seine, served the purpose to present the spatial structure of town up to unbelievable precision. The arrow directs to transversal of the city, going from Louvre, over Concord Square and Triumph Gate up to new Grande Arche de la Defense.

There are architectural facilities around the world, which represent traditional landmarks and as such they are accepted in mass culture and suggested to the individual. Paris, which many people connect with Eiffel Tower, just like Athens is connected with Parthenon, or Sydney Opera House with that city, are the first things that come to people's minds, even though they have never been there. However, when they reach Sydney for instance, they will search the famous opera house there.

If we make analyses of historical position of facility built vertically from the position of meaning in our consciousness, based on previous, we can find following reasons for their building:

- Vertical facility (or element) as the landmark in space

- Vertical facility created for praising God and aiming to the sky

- Vertical facility as a manifestation of economical and technological power

- Vertical facility as beneficial element - economical category 

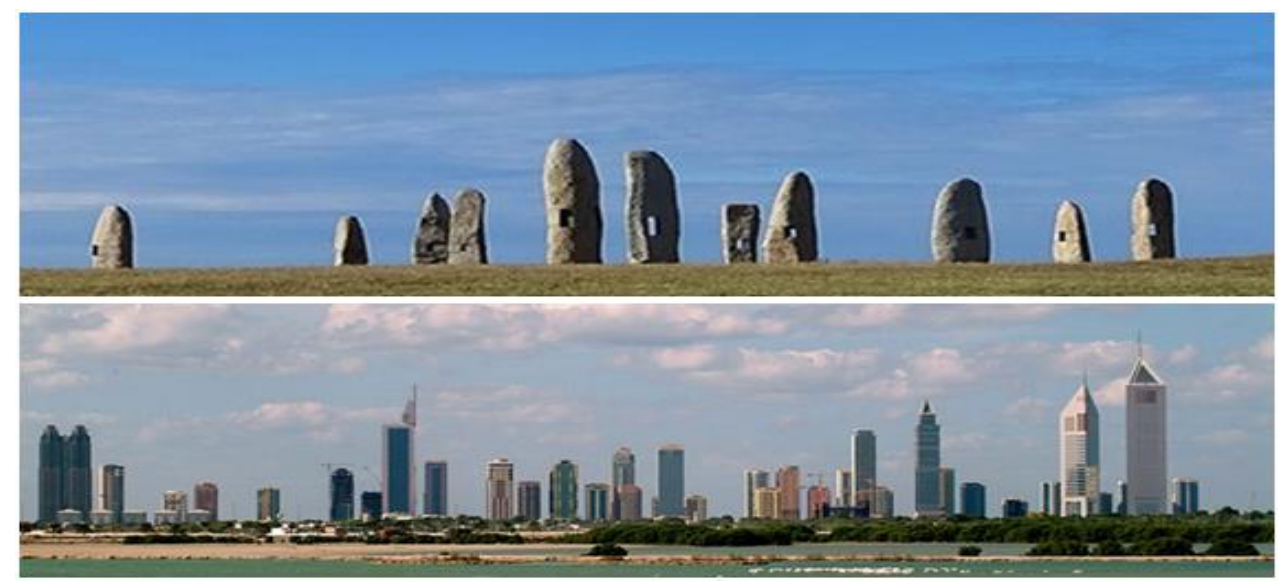

Fig.8. No matter the time of origin, landmarks created by vertical building have similar or the same role

What can be concluded, based on previous observations, is the fact that vertical facility is historically constant with its effect on perception and beholder's experience. Reasons for vertical building were changing throughout the history (from location in space and positioning, to religious, utilitarian, culturological, engineering etc.) and by that, facilities have various meanings but only one constant: they are landmarks and represent one of the most significant creative meand of the urban image. So, verticals are material required for successfully formation of urban picture and orientation in space (Fig.8).

An important element represents their meaning and symbolical image (created during the time) in space. That factor is one of the most important elements of cognitive processes taking place in human brain. [15] The importance of the facility in the moment of perceiving and through the history represents basic material for positioning of each object in human perception and memory. Therefore, previous information about observed significantly contribute to cognitive processes.

The abundance of meaning and respecting the beholder to urban vertical is not the same, having in mind function, meaning and the purpose of the facility.

Without any doubt, the highest value through the history of city development, had religious buildings, so cities, nations and countries identified themselves with such facilities. Through 19th, 20th and 21st century, high buildings dedicated to multinational companies are also signs of a significant place in urban, country, and even in world surrounding (Sydney Opera House, Eiffel Tower, Big Ben, etc.).

Nowadays, cultural facilities are aiming upwards, so they also become significant landmarks with one element, proved to be dominant in space: height (Fig.9).

Not only the case of Philcharmonic in Hamburg can prove that the height is an important attribute of perceiving space, adding some significance to other facilities on that particular place. That is not a wonder, because it is clear that architectures of culture and sport in 20th and 21 st century surpress religious facilities. 

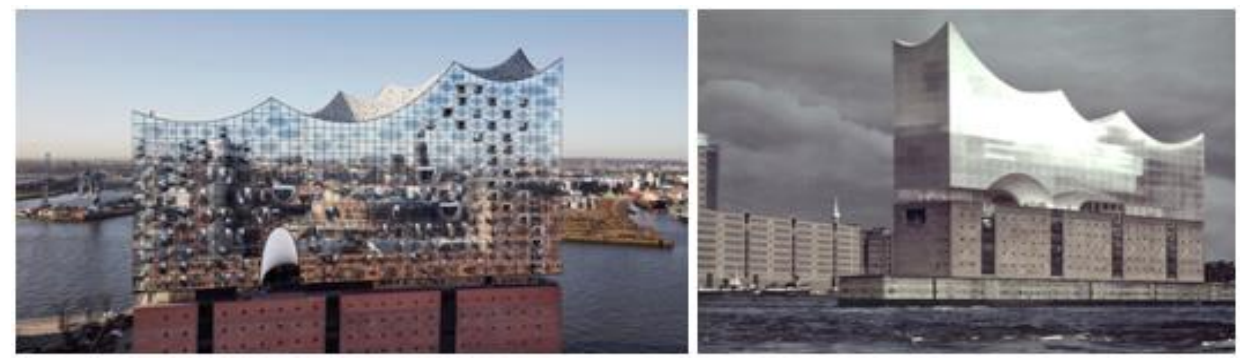

Fig.9. Filcharmony in Hamburg. Architects: Herzog \& de Meuron.

For the last 100 years, vertical facilities in contemporary historical moment and developing of the city are not in such a good position because of mutual ruthless race for space overruling to show the power of multinational companies. [16]

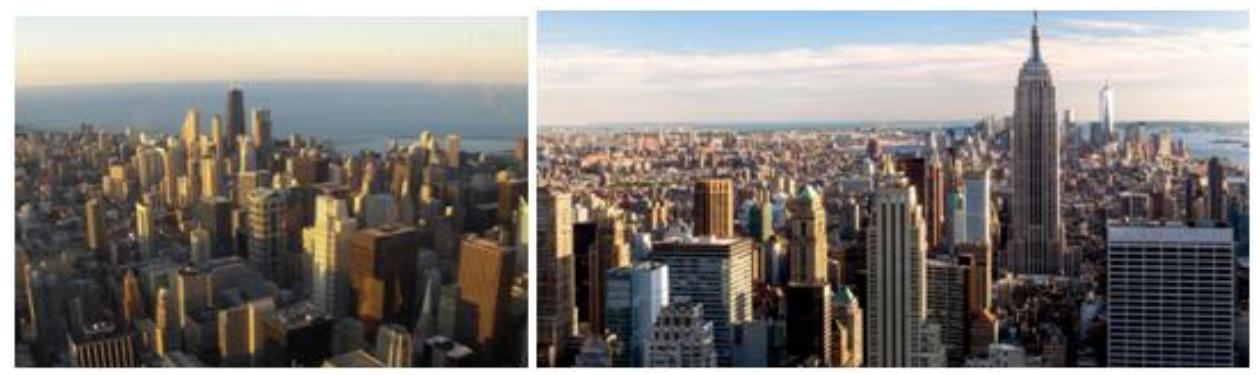

Fig.10. Uncontrolled race of vertical buildings destroys hierarchy of urban core of modern cities.

That speaks volumes about negative appearance manifested in disturbing, previously clear, spatial hierarchy. That is no more the church tower or the mosque tower, but a multinational company building which can be endangered by another more powerful company within a year (Fig.10).

That is why vertical facilities need to regain their position recommended by historical experience. Especially for reason of it being a powerful tool in creating spatial hierarchy and meaning carrying.

\section{References}

1. A. Geremek, M. Greenlee, S. Magnussen Perception Beyond Gestalt. (Psihology Press. London and New York, 2014)

2. R. Arnheimm Art and Visual Perception. (University of California Press. Berkley, New York, London, 1974)

3. W. R. Crozier, A. J. Chapman Cognitive Processes in the Perception of Art. NorthHolland (Amsterdam, New York, Oxford)

4. R. Arnheim The Dynamics of Architectural Form. University of California Press. Berkeley, (Los Angeles, London, 1977)

5. G. Bachelard The Poetics of Space. Book on Demand. (2012)

6. J. D. Borstin The Creators. Random House. (1982)

7. K. Linch The Image of the City. The M:I:T Press., Massachusettc Insdtitute of Technology, Cambridge, Massachusettc. (1972) 
8. A. Ballantyne, Architecture: A Very Short Introduction. Oxford University Press. (2005)

9. R. Arnheimm The Power of the Center. (University of California Press. Berkley, New York, London, 1982)

10. R. Arnheimm The Power of the Center. (University of California Press. Berkley, New York, London, 1985)

11. K. Lewin A Dynamic Theory of Personality. McGraw-Hill.(1967)

12. M. Eliade The Sacred and The Profan. A Harvest Book. Harcourt, Inc. (1957)

13. R. Arnheimm Visual Thinking. University of California Press. Berkley, New York, London. (1997)

14. R. Venturi Complexity and Contradiction in Architecture. The Museum of Modern Art, New York (1977)

15. S. Pinker How the Mind Works. W. W. Norton Company. (1999)

16. R. Koolhaas, B. Mau $S, M, L, X L$. Monacelli Press (1997) 